\title{
Medical Errors in a Newly Established Teaching Hospital- Some Lessons Learnt for Better Practices
}

\author{
Nida Anwarr,*, Haya Ul Mujtaba ${ }^{2}$, Naveena Fatima², Abdul Latif ${ }^{3}$, Tahir Sultan Shamsi ${ }^{1}$ \\ ${ }^{\prime}$ Department of Clinical Hematology \& Administration, National Institute of Blood Disease \& Bone Marrow Transplantation, Karachi, Pakistan. \\ ${ }^{2}$ Department of Research \& Development, National Institute of Blood Disease \& Bone Marrow Transplantation, Karachi, Pakistan. \\ ${ }^{3}$ Department of Administration, National Institute of Blood Disease \& Bone Marrow Transplantation, Karachi, Pakistan.
}

\begin{abstract}
Objective: Medical errors (MEs) are flaws in implementation of act and failure of planning associated with patients. To avoid errors healthcare units are trying to make safer strategies that decrease morbidity and mortality due to MEs. The aim of this study was to evaluate errors and make strategies to avoid such errors in future.
\end{abstract}

Methods: A cross sectional study conducted at NIBD-PECHS campus Karachi, Pakistan. Approval was taken from institutional review board. Data was collected from February 2018 to January 2019. Reporting form included variables like reporting month, location, department, classification, root cause, risk, action taken, financial burden and status. Analysis was done by using SPSS 23.0.

Results: A total of 42 MEs were reported at our hospital which were divided into four categories: patient care events 19 (45.2\%), management events 17 (40.5\%), criminal events 3 (7.1\%) and equipment error errors 3 (7.1\%). Most of errors occurred in inpatient department 27 (64.2\%) and nurses were responsible for $17(40.5 \%)$ errors. In most of cases $23(54.8 \%)$ root cause was staff negligence and to resolve this 22 (52.4\%) verbal warnings were given. About 367.62USD (52,000PKR) were consumed to resolve the errors.

Conclusion: We concluded that reporting MEs is practical approach to give quality services to patients and facilitates in making new policies to reduce errors in future.

Keywords: Medical errors, Reporting, Management, Healthcare structure, Hospital system, Protocols, Hospital policies, Hospital practices.

\section{INTRODUCTION}

"Error" is a simple word that means "mistake". When it's used with the word medical its meaning becomes more complex. The simple mere definition of the term medical error does not exist and hence complexly this is defined as an unintentional catastrophe (either of omission or commission) or one that does not accomplish its expected outcome. It may also mean facing the disappointment of a planned activity to be finished as per anticipation (an error of the implement of the act) or wrong plan of action to complete the intend (an error of planning) or a divergence from the method of care that plans in the betterment of a patient's health. Medical errors cause harm to the patients due to failure of the system or at the individual level [1]. On daily basis, a number of people in the world utilize health care services. It is thus duty of health care units to provide good and quality care to patients. But unfortunately the medical errors (MEs) are reported globally every day [2]. Common MEs that occur in the health care organizations are medicine related events, transfusion reactions, wrong diagnosis, incident during surgery like excessive bleeding, accidental injuries, wrong patient identification or failure to apply infection control measures. The high risk MEs occur most often in intensive care units, operation theaters and emergency departments [3]. These events may vary from minor injuries to death of patients.

\footnotetext{
*Address correspondence to this author at the Department of Clinical Hematology \& Administration, National Institute of Blood Disease \& Bone Marrow Transplantation, Karachi, Pakistan. E-mail: drnidairfan@yahoo.com
}

A Survey conducted by European commission in 2005, reported that $23 \%$ of European population had been facing medical error personally or by family members in which $11 \%$ of medical errors were wrong prescribed medications [4]. In 2015, a report was published by institute of medicine publication, "To Err is Human" in which they reported 98,000 people die yearly due to the medical errors $[5,6]$. On regional level, about 4.4 million MEs occur yearly in hospitals of developing countries of eastern Mediterranean region [7]. In hospitals of Iran the highest frequency of MEs are systemic error, administrative error and adverse drug events. In Iran some clinical supervisors declared out of 100 to 150 patients, 01 patient die due to ME [8]. MEs not only cause of harm to patients but also implicate a great financial burden [9]. In 1999, a study conducted in the US revealed estimated total cost of about \$19-\$27 billion that was spent on prevention of MEs annually [10]. A number of cases of MEs are unreported which is the leading cause of morbidity and mortality worldwide. Reporting is one of the approaches to minimize the number of errors. Hence, reporting MEs considerably affect on patient's wellbeing. Charles Billings, originator of the aviation safety reporting system in the US, pinpointed that the two major aspects that are the barrier in reporting MEs are fright of punishment or embarrassment and faith that reporting leads to betterment [11]. There seems to be biasness in reporting MEs, specialist would report if they feel safe and secure or would avoid reporting because of being sued, penalized and legal threat to their medical license [12]. Reporting of MEs is supposed to be 
obligatory in hospitals. It is the first key step in terms of prevention of patients' morbidity and mortality [13].

Pakistan is an underdeveloped country in which the rate of MEs is high due to under supervised and unvigilant medical systems. Moreover medical literature in this context is scant. A number of cases of medical flaws are reported in Pakistan such as wrong diagnosis, usage of expire medicine, administration of wrong oral medicines and injections, leaving of instruments in abdomen during operations, wrong treatment giving that led to severe complications to death of patients [14]. MEs are the eighth biggest cause of deaths in our country. According to a statistics approximately 7,000 people die per year due to MEs. A number of causes are accountable for the occurrence of these MEs. Pakistan medical and dental council (PMDC) is an organization that is responsible for making laws and regulations regarding the health care in Pakistan. But unfortunately our health care systems fail to execute these laws and regulations. Infrastructure of hospitals lacking the trained staff, burden on staff due to work load, extra duty hours, corruption in hospitals are the most important highlighted factors that are associated with occurrence of MEs [13]. To overcome these problems health care organizations should make strategies to overcome and prevent MEs in order to give good and quality care to the patients [15].

Thus with this rational, our study was conducted to identify the flaws in newly established hospital system, observe the fear that is the barrier in reporting, and make protocols to avoid further errors and also to add data in local literature.

\section{MATERIALS AND METHODS}

This cross sectional study was conducted at NIBD-PECHS campus Karachi, Pakistan. Approval was taken from institutional review board before starting the study. We collected the data of all MEs related to the patient's treatment outcome as well as management side which were reported from February 2018 to January 2019. A pre designed MEs reporting form was formed and filled including variables like: ME reporting date, location, department involved, staff involved, classification of ME, root cause, risk, actions taken, implicated financial burden, status after taking all measures. It was made mandatory as per policy to fill this form after the occurrence of any ME at the hospital. These forms were filled by the person who was responsible for ME and submitted to concerned departmental incharge and then higher management to take preventable measure(s) to stop that event and steps taken to stop to avoid such events in future. On the other hand we arranged weekly teaching classes and follow up meetings for all the departments and trained our staff accordingly. Guidelines issued by University of Kwazulu-Natal for MEs reporting were followed and thus adverse events were classified into 5 categories based on risk stratification i.e. insignificant errors not involving patient or financial loss, minor risk events that were treated without patient harm and minor financial loss, moderate risk events that were source of less patient harm and medium financial burden, major risk events that directly caused harm on treatment outcome and cause of large financial burden while the last was catastrophic events that cause death, financial loss or danger for goodwill of institute [3]. Data was entered by using Microsoft Excel. Analysis was done on SPSS version 23.0. Frequencies and percentages were calculated for qualitative data.

\section{RESULTS}

A total of 42 MEs were reported from February 2018 to January 2019 at our hospital. Out of total, maximum number of errors i.e. $27(64.2 \%)$ occurred at the inpatient department while the others occurred at blood donor area, emergency department, reception, laboratory and operation theater with the frequency of $06(14.2 \%), 01(2.2 \%), 02$ (4.6\%), 05 (12\%) and $01(2.2 \%)$ respectively (Fig. 1). Out of total MEs, high frequency of errors occurred due to the negligence of nurses $17(40.4 \%)$, laboratory technicians $12(28.6 \%)$ followed by doctors $05(12 \%)$, pharmacists $04(9.5 \%)$, unit receptionists $03(7.1 \%)$ and housekeeping staff $1(2.4 \%)$. The frequency of MEs was much higher in the initial 6 months phase i.e., 41 $(97.6 \%)$ as compared to later phase i.e., $1(02.4 \%)$. MEs were divided into four categories as shown in Table 1. Management events included all events that occurred due to the mismanagement at administrative side, criminal events included unethical issues, patient care events included errors that occurred in terms of treatment provision while the equipment error events comprised of issues that occurred due to system or machine error.

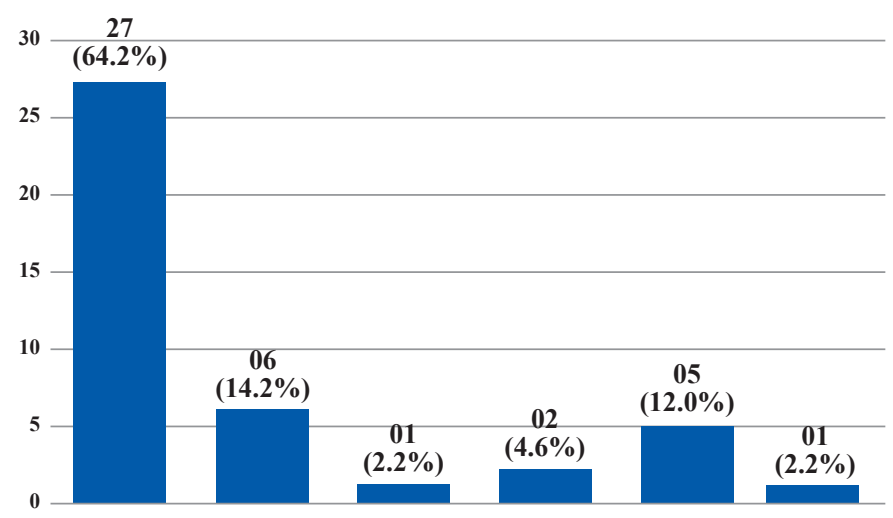

Fig. (1). Frequency of Medical Errors at Department Level.

Based on risks stratification, errors were classified into three groups. Out of 42 errors 30 (71.4\%) were high risk, 06 $(14.3 \%)$ were moderate risk, although other $06(14.3 \%)$ were low risk (Fig. 2). We also investigated the root cause of the MEs that occurred at our hospital. The distribution of root causes along with their frequencies and percentages (\%) are shown in Table 2 . We effectively solved and identified the root cause of $40(95.2 \%)$ events while $02(4.8 \%)$ events remain unsolved but according to root cause we categorized those events into staff negligence. 


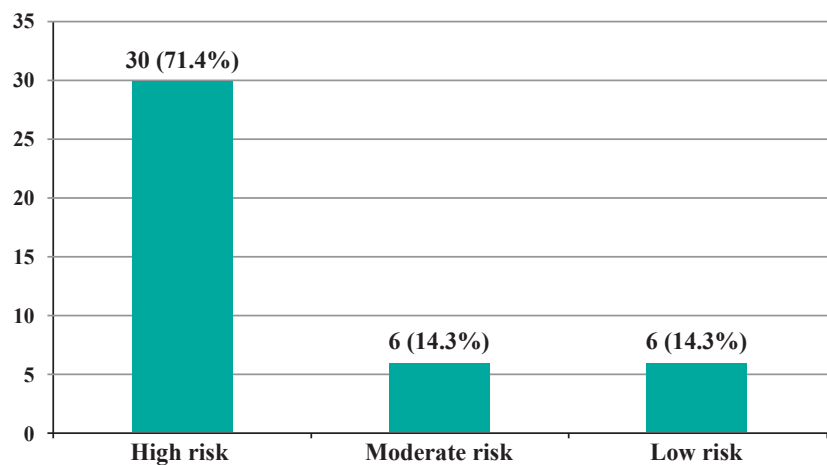

Fig. (2). Frequency of Risk Stratification of MEs.

After the MEs occurred, management investigated the errors and measures were taken to resolve and avoid such errors in future. Actions were taken against the involved personal(s) or department incharges that were directly or indirectly involved in occurrence of MEs. Actions that were taken along with their frequencies and percentages (\%) are depicted in (Table 3).

These MEs were also a source of the financial burden on the institute. In our hospital out of total MEs about $29(69 \%)$ had no financial loss while 13(31\%) implicated financial loss. Approximately the amount of Rs.52,000 is equivalent to 367.62 USD was spent on fixing MEs at our hospital.

Table 1. Frequencies and Percentages of Categories of Medical Errors.

\begin{tabular}{|l|c|c|}
\hline Category of MEs & Frequency & Percentage (\%) \\
\hline Patient care events & 19 & 45.2 \\
\hline Management events & 17 & 40.5 \\
\hline Criminal events & 03 & 7.1 \\
\hline Equipment error events & 03 & 7.1 \\
\hline
\end{tabular}

Table 2. Frequencies and Percentage of Root Causes of Medical Errors.

\begin{tabular}{|l|c|c|}
\hline Root Causes & Frequency & Percentage (\%) \\
\hline Staff negligence & 23 & 54.8 \\
\hline Communication gap & 07 & 16.7 \\
\hline Lack of knowledge & 06 & 14.3 \\
\hline Attitude of staff & 03 & 4.8 \\
\hline Problem in equipments & 02 & 4.8 \\
\hline Leadership failure & 01 & 2.4 \\
\hline
\end{tabular}

Table 3. Frequencies and Percentage of Root Causes of Medical Errors.

\begin{tabular}{|l|c|c|}
\hline Actions Taken & Frequency & Percentage (\%) \\
\hline Verbal warning & 22 & 52.4 \\
\hline Warning letter issued & 07 & 16.7 \\
\hline Suspension from duty & 03 & 7.1 \\
\hline Shifted duty to another bench & 03 & 7.1 \\
\hline Object replaced & 02 & 4.8 \\
\hline Extra duty hours & 01 & 2.4 \\
\hline Equipment changed & 01 & 2.4 \\
\hline Patient refunded & 01 & 2.4 \\
\hline Responsibilities changed & 01 & 2.4 \\
\hline Stop transfusion & 01 & 2.4 \\
\hline
\end{tabular}

\section{DISCUSSION}

Medical errors are one of the leading causes of death worldwide. Reducing MEs and increasing patient safety is one of the main targets of any health care organization [16], and reporting is an essential tool to prevent MEs [4]. Our hospital was newly established system where we made an attempt to execute the system of reporting by designing a special medical error reporting form in order to minimize MEs that are directly or indirectly associated with morbidity and mortality. As we were focusing towards the betterment we divided our study in two phases to observe the difference initial and later phase. In 2017, a study was conducted at Nigeria which concluded the incidence of $42 \%$ MEs [17]. In our study we found $97.6 \%$ MEs in initial 06 months of hospital establishment and only $2.4 \%$ occur in later 6 months. However, we could not find a study done in context of comparison between initial and later phases. The reasons of high percentage of MEs in initial months could be less familiarity with policies, rules and regulations of newly appointed staff. As we conducted training sessions and teaching classes' reduction of MEs were seen in later phase. A study conducted at US reported high percentage of MEs i.e. 19\% in inpatient department particularly treatment related MEs [18, 19]. However, in our study we found $64 \%$ MEs in inpatient department including wrong test indent, wrong dispense of medicine, contaminated blood product issuance, less adherence with the protocol of discarding hazardous wastes and lack of knowledge regarding transfusion reactions. In a European study $0.15 \%$ errors were reported at blood donor area [20]. This is in contrary with our study findings where $14 \%$ errors were reported at blood donor area. Contrary findings were also reported in terms of errors in clinical laboratory i.e. we found $12 \%$ error in clinical laboratory whereas in another study from Pakistan, only $1.2 \%$ errors were reported [21]. In emergency department $2.2 \%$ errors were reported in our study. Similar findings were observed by an Iranian study reporting $1.24 \%$ errors in emergency department [22]. In 2004 a study was conducted at Maryland in which they stated the frequency of errors done at physician and nursing end and that was $24 \%$ and $54 \%$ [23], which is higher than the percentage found in our study i.e. $12 \%$ and $17 \%$ respectively. The reason for high percentage of errors in nursing department could be lack of trained nursing staff. According to national quality forum standard [24], we divided events into 6 categories out of which we found data for only 04 categories. According to our study $45.2 \%, 40.5,7.1 \%$ and $7.1 \%$ were related to patient care events, management events, criminal events and equipment error events. We did not have the running operation theater and only a single incidence was reported. Thus we included that error in management event. None of the errors were reported in environmental category. As per risk management categorization, we found $71.4 \%$ high risk events which is somewhat comparable to the major risk events identified at US [25]. Financial cost that used to resolve MEs in our study was USD 367.62 (PKR 52,000) for 
13 (31\%) events in one year. It was a single institute based study. In US around \$19-\$27 billion are used in prevention of MEs per year [10].

We took measures to resolve MEs (Table 3) such as issuance of warning letter, responsibilities change, and suspension from duty, verbal warning and stop transfusion in transfusion reactions. In a study of Maryland, actions taken as a result of the errors included verbal warning (26\%), enhancing communication (26\%), and providing additional training (12\%) [23]. We also emphasized on training by conducting workshops, lectures and hands on practices to avoid MEs. We successfully resolved $95.2 \%$ cases while the $2.8 \%$ cases were remained unsolved. Unresolved errors were classified as staff negligence and verbal warning was given to all concerned staff.

\section{CONCLUSION}

We concluded that reporting MEs is a practical approach to promote safety and provision of quality services. It also facilitates making new strategies and policies in term of minimizing the rate of errors. Now-a-days ongoing research is being conducted to formulate the local policies and execution of best practices. This study would give an insight on how to cope up with the MEs by reporting and thereby implementing of policies that will ultimately give benefit to patients and healthcare facility.

\section{FUNDS FOR STUDY}

This study was not funded.

\section{CONFLICT OF INTEREST}

Declared none.

\section{ACKNOWLEDGEMENTS}

We acknowledge our administration staff for their cooperation and assistance during this study.

\section{REFERENCES}

[1] Dovey S, Phillips R. What should we report to medical error reporting systems? BMJ 2004; 13(5): 322-23.

DOI: $10.1136 /$ qshc.2004.011791

[2] Technical Series on Safer Primary Care. Geneva: World Health Organization 2016; License: CC BY-NC-SA 3.0 IGO. Available at : https://www.who.int/patientsafety/topics/primary-care/technical_series/en/

[3] Mehomed O. Adverse events monitoring and reporting guidelines. Durban, South Africa: University of Kwa-Zulu Natal. 2002; Available at: http://www.kznhealth.gov.za/family/Adverse-events-monitoring-reporting-guidelines.pdf

[4] European Commission (EC). Medical Errors. Special Eurobarometer 2006; Available at: https://ec.europa.eu/commfrontoffice/publicopinion/archives/ebs/ebs_241_en.pdf
[5] Harolds JA. Quality and safety in health care, part III: To Err is human. Clin Nucl Med 2015; 40(10): 793-5. DOI: $10.1097 /$ RLU.0000000000000878

[6] Thomas EJ, Studdert DM, Burstin HR, et al. Incidence and types of adverse events and negligent care in Utah and Colorado. Med Care 2000; 38(3): 261-71.

DOI: 10.1097/00005650-200003000-00003

[7] Vaziri S, Khansari P, Mansouri F, et al. Frequency and types of medical errors in infectious patients referred to the emergency department of Imam Reza (AS) Hospital in Kermanshah, Iran (2014- 2015). Glob J Qual Saf Healthcare 2016; 4(4): 455-9.

[8] Khammarnia M, Setoodehzadeh F. Medical Error as a Challenge in Iran's Health System. Health Scope 2017; 6(1): in press. DOI: $10.17795 /$ jhealthscope-39743

[9] Urbach DR, Govindarajan A, Saskin R, Wilton AS, Baxter NN. Introduction of surgical safety checklists in Ontario, Canada. N Engl J Med 2014; 370(11): 1029- 38.

DOI: $10.1056 /$ NEJMsa1308261

[10] Thomas EJ, Studdert DM, Newhouse JP, Zbar BI. Costs of medical injuries in Utah and Colorado. Inquiry 1999; 36(3): $255-64$.

[11] Billings CE. Some hopes and concerns regarding medical event-reporting systems: Lessons from the NASA aviation safety reporting system. Arch Pathol Lab Med 1998;122(3): 214.

[12] Cohen MR. Why error reporting systems should be voluntary: They provide better information for reducing errors. Brit Med J 2000; 320(7237): 728-9. DOI: 10.1136/bmj.320.7237.728

[13] Gadit AA. Medical errors: Who is to be blamed? J Pak Med Assoc 2012; 62(4): 406-7.

[14] Shiwani MH, Gadit A. Medical negligence: A growing problem in Pakistan. J Pak Med Assoc 2011; 61(6): 610-1.

[15] Hut-Mossel L, Welker G, Ahaus K, Gans R. Understanding how and why audits work: protocol for a realist review of audit programmes to improve hospital care. BMJ Open 2017; 7(6): e015121. DOI: 10.1136/bmjopen-2016-015121

[16] Institute of Medicine, (US) Committee on Quality of Health Care in America. Crossing the quality chasm: A new health system for the 21st century. Washington DC: Academic Press 2001; Report No.: 0309072808.

[17] Iloh GUP, Chuku A, Amadi AN. Medical errors in Nigeria: A cross-sectional study of medical practitioners in Abia State. Arch Med Res 2017; 5(1): 44.

[18] Forster AJ, Clark HD, Menard A, et al. Adverse events among medical patients after discharge from hospital. CMAJ 2004; 170(3): 345-9. 
[19] Forster AJ, Murff HJ, Peterson JF, Gandhi TK, Bates DW. The incidence and severity of adverse events affecting patients after discharge from the hospital. Ann Intern Med 2003; 138(3): 161-7.

DOI: $10.7326 / 0003-4819-138-3-200302040-00007$

[20] Das SS, Chakrabarty R, Zaman R. Monitoring errors in a blood bank immunohematology laboratory: Implementing strategies for safe blood transfusion. Glob J Transfus Med 2017; 2(2): 118. DOI: 10.4103/GJTM.GJTM_40_17

[21] Sadiq F, Yasmeen F, Mumtaz A, et al. Frequency of errors in clinical laboratory practice. Iran J Path 2014; 9(1): 45-9.

[22] Asadi P, Modirian E, Dadashpour N. Medical Errors in Emergency Department; a Letter to Editor. Arch Acad Emerg Med 2018; 6(1): 33.
[23] Pham JC, Story JL, Hicks RW, et al. National study on the frequency, types, causes, and consequences of voluntarily reported emergency department medication errors. J Emerg Med 2011; 40(5): 485-92.

DOI: 10.1016/j.jemermed.2008.02.059

[24] Serious reportable events in healthcare: A consensus report. Washington, DC: National Quality Forum 2002.

[25] Kaldjian LC, Jones EW, Wu BJ, Forman-Hoffman VL, Levi $\mathrm{BH}$, Rosenthal GE. Reporting medical errors to improve patient safety: A survey of physicians in teaching hospitals. Arch Intern Med 2008; 168(1): 40-6.

DOI: 10.1001/archinternmed.2007.12

(C) 2019 National Journal of Health Sciences.

This is an open-access article. 